\title{
Embedding Physical Activity Within Community Home Support Services for Older Adults in Ireland - A Qualitative Study of Barriers and Enablers
}

\author{
Lauren Swan ${ }^{1,2}$, N Frances Horgan ${ }^{3}$, Vanda Cummins ${ }^{4}$, Elissa Burton $\mathbb{D}^{5}$, Rose Galvin ${ }^{6}$, \\ Dawn A Skelton ${ }^{7,8}$, Bex Townley ${ }^{8}$, Frank Doyle $\mathbb{D}^{9}$, Samira B Jabakhanji ${ }^{10}$, Jan Sorensen $\mathbb{D}^{10}$, \\ Debbie Rooney², Lisa Murphy², Austin Warters ', Maria O'Sullivan' \\ 'Department of Clinical Medicine, Trinity College Dublin, Dublin, Ireland; ${ }^{2}$ North Dublin Home Care (NDHC), Dublin, \\ Ireland; ${ }^{3}$ School of Physiotherapy, RCSI University of Medicine and Health Sciences, Dublin, Ireland; ${ }^{4}$ Primary Care \\ Physiotherapy Services CHO9, Health Service Executive (HSE), Dublin, Ireland; ${ }^{5}$ Curtin School of Allied Health, Curtin \\ University, Perth, Australia; ${ }^{6}$ School of Allied Health, Ageing Research Centre, University of Limerick (UL), Limerick, \\ Ireland; ${ }^{7}$ School of Health and Life Sciences, Glasgow Caledonian University, Glasgow, Scotland; ${ }^{8}$ Later Life Training, Killin, \\ UK; ${ }^{9}$ Department of Health Psychology, RCSI University of Medicine and Health Sciences, Dublin, Ireland; ${ }^{10} \mathrm{Healthcare}$ \\ Outcomes Research Centre (HORC), RCSI University of Medicine and Health Sciences, Dublin, Ireland; "Older Person \\ Services $\mathrm{CHO}$, Health Service Executive (HSE), Dublin, Ireland
}

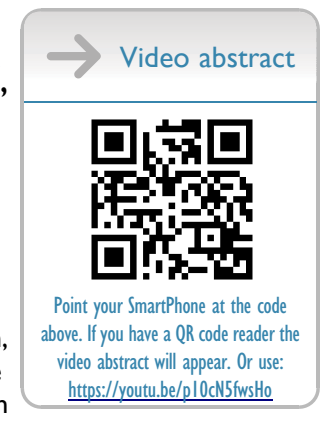

Correspondence: Maria O’Sullivan, Email maria.osullivan@tcd.ie

Introduction: In Ireland, over 55,000 older adults are supported in their community by formal home support, amounting to an estimated 23 million care hours annually. There is a growing need to move beyond care, to more proactive approaches to maintain physical function. In a feasibility study, we delivered the "Care to Move" (CTM) program through existing home support services. This qualitative study aimed to explore the experience and perceptions of Health Care Assistants (HCAs), who were trained in and delivered the CTM program.

Methods: We conducted semi-structured telephone interviews with 22 HCAs [mean age $49.0 \pm 10.7$ years and female 21/22] involved in the delivery of the program with older adults $[n=35$, mean age 82.8 (7.8) years]. Interview transcripts were coded and analyzed thematically to capture barriers and enablers to program delivery.

Results: Barriers and enablers were identified under three themes i) the CTM approach ii) the home support setting, iii) older adults and physical activity, with iv) delivering care in a crisis and v) future directions further identified. Overall, there was a positive perception of the program's focus on "movement prompts and motivators", the "fit" within home support services, and the training provided. Practical challenges of limited time and the task-orientated nature of home support were reported as recurring barriers for CTM. Many HCAs commented on the value and perceived positive benefits of the program for their clients. Though negative perceptions of older adults' motivation or ability to engage with physical activity were noted. Risk, such as injury or pain, was identified but was not a dominant theme.

Conclusion: Our findings suggest that embedding physical activity initiatives within home support services could be feasible. Restructuring of services, engaging HCAs, and moving beyond traditional "task-oriented" care models to more personalised proactive approaches may facilitate this initiative and support aging in place.

Keywords: aging, physical activity, integrated care, frailty, aging in place

\section{Introduction}

Globally, an estimated 1.4 billion people will be aged over 60 years by $2030 .{ }^{1}$ An aging global population has fueled a shift in policy towards models of care that support aging in place. Given the impact of the COVID-19 pandemic on long-term residential care and the well-documented preference of individuals to remain living at home, enhancing community-based 
care services is an emergent priority. ${ }^{2,3}$ In 2021, an estimated 23.9 million hours of government-funded home support services (termed domiciliary care in some regions) were delivered to over 55,000 people aged 65 years and older in Ireland. ${ }^{4}$ State-funded home support encompasses assistance with activities of daily living (ADLs), most commonly personal care ${ }^{5}$ and is allocated based on a needs assessment, and is currently not income assessed in Ireland. This group of older people may be considered to be on a boundary between community-based and long-term residential care, with $8 \%$ transitioning to longterm residential care annually. ${ }^{6}$ This trajectory is not however inevitable, and evidence supports measures to enable aging in place, in terms of both the desires and wishes of older people and the cost savings in delivering care. ${ }^{7}$

Previous studies, utilizing administrative data, characterize older adults receiving home support as of advanced age (mean age 83 years), living alone, with a high prevalence of dementia (37\%) and frailty $(42 \%)$, ${ }^{6,8,9}$ who are often underrepresented in research. Frailty refers to the gradual loss of in-built reserves giving rise to vulnerability. ${ }^{10}$ Older adults with frailty have a higher prevalence of sedentary behavior and may avoid physical activity due in part, to risk aversion. ${ }^{11,12}$ Recent guidelines, however, recommend physical activity in older age, specifically to reverse or delay the onset of frailty. ${ }^{13,14}$ It is suggested that physical activity may benefit conditions without effective pharmacological treatment, such as dementia and sarcopenia, with an aim to supporting older adults to remain living at home for longer. ${ }^{15}$ Recent evidence supports the importance of light intensity activity to health, a message that is particularly important to communicate to those who are currently inactive and/or frailer. ${ }^{16,17}$

The majority of structured exercise programs are delivered by allied health professionals and outside of the home, for example in community health centers. ${ }^{18}$ Commonly cited barriers by older adults to engaging in community-based physical activity programs include subjective poor health and a lack of transport. ${ }^{19}$ Furthermore, older adults in receipt of formal home support services have expressed a preference for being physically active through the activities they enjoy, such as walking and gardening, rather than engaging in structured exercise programs. ${ }^{20}$ Consistent with this, frail older adults have been shown to benefit from home-based one-to-one approaches aimed at maintaining physical function and more structured exercise performed at home. ${ }^{21-23}$ An estimated 40,000 Health Care Assistants (HCAs), are employed in the delivery of formal home-based care in Ireland, primarily assisting people with ADLs and personal care. ${ }^{24}$ "HCA" is used in Irish healthcare, though, several other terms are in use nationally and internationally in practice and research to describe the staff who participated in this study (including home care worker, home care assistant, home help, caregiver, home support worker, domiciliary care worker). Engaging HCAs delivering formal home support services, may provide a potential pathway to promote physical activity and retention in home-based programs due to their frequent and sustained contact with older adults. ${ }^{25}$ Furthermore, greater experience and positive ageing attitudes among community care staff were reported as enablers to the delivery of a falls prevention program within home care services. ${ }^{25}$

This work forms part of the feasibility study of embedding the Care to Move (CTM) program into a care at home setting. ${ }^{26,27}$ While Care To Move is referred to as a program throughout, it is not a structured exercise plan. ${ }^{26,27}$ Care to Move is a whole workforce-based approach that aims to bring consistent language to the promotion of "movement" within formal care services. ${ }^{26}$ This approach emphasizes "movement prompts" that encourage older adults to "sit less and move more". The experience and acceptability of delivering the program, including "movement prompts" by HCAs to their home support clients, however, is not known. Therefore, this qualitative study aims to examine the HCA-perceived barriers and enablers of embedding physical activity approaches within formal home support services. The overall goal was to better understand the feasibility of translating this initiative into practice. It is anticipated that the findings will assist the design of evidence-based care through proactive approaches to support aging in place.

\section{Methods}

\section{Study Design and Data Collection}

In this descriptive qualitative study, ${ }^{28}$ we conducted semi-structured telephone interviews with HCAs who were trained in CTM and subsequently encouraged and supported movement in daily life tasks with older adults in receipt of formal home support services. The primary feasibility study and the CTM approach are described in detail elsewhere. ${ }^{26,27}$ In brief, the physical activity initiative was embedded into existing formal home support services and delivered by HCAs. Prior to delivering the initiative, HCAs completed a 2-day CTM training course delivered by a Senior Physiotherapist 
and qualified CTM trainer. The CTM training included communicating the importance of movement, deploying specific movement prompts within usual care activities, and motivating older adults to engage with previously prescribed home exercise plans designed by formal therapy services. The clients in receipt of the program $(n=35)$ were of mean age 82.8 \pm 7.8 years, with mild to moderate frailty $(88.5 \%, \mathrm{n}=31 / 35)$ as indicated by the clinical frailty scale. ${ }^{10}$

The semi-structured interviews explored the HCAs' experiences and views of promoting physical activity, barriers, and enablers to the CTM approach, delivery of the program and suggestions for the future (Appendix 1). The latter included views on the potential role of technology. All interviews were conducted by telephone (LS and FH) between August and September 2020, audio-recorded, and transcribed verbatim. The average length of interview was 16 minutes (range from 11 to 24 minutes). The study was approved by the Royal College of Surgeons in Ireland Research Ethics Committee (REC - 2018:1489). Written informed consent was obtained from all participants which included publication of anonymized responses. Accordingly, HCAs are described using coded identification numbers (eg HCA001).

\section{Data Analysis}

All transcripts were analyzed using a reflexive approach to thematic analysis which followed the 6 phase guide provided by Braun and Clarke to identify themes within the data. ${ }^{29,30}$ Interviews were read in their entirety by research team members LS and MOS. Following this, inductive coding commenced, wherein LS and MOS systematically highlighted segments of data which included words and phrases relevant to each code. ${ }^{31}$ Codes with similar meaning were grouped and collapsed to form themes pertinent to enablers and barriers to implementation of the program. These themes were then reviewed by researcher AW who conferred with LS and MOS to discuss overarching themes. Following refinement of the identified themes, other authors were consulted for feedback and consensus based on the interview data, ensuring that over-arching themes and sub-themes accurately reflected the supporting data. Finally, all authors provided feedback on the written manuscript. Experienced qualitative researchers FH and LS conducted all semi-structured interviews and both research team members MOS and AW had experience in qualitative data analysis. Authors who conducted the analyses did not partake in the CTM training delivered to the HCAs, to maintain objectivity and reduce bias.

\section{Results}

In total, 22 HCAs participated in the study interviews and were predominantly female (21/22) with a mean age of $49.0 \pm$ 10.7 years. The group were experienced in care provision for older adults, having been employed in their current organization for an average of $10.5 \pm 6.5$ years (range 1.4 to 20.7 years). HCAs delivered CTM for a minimum of seven months at the time of interview. Perceived barriers and enablers to the delivery of the program within home support were identified across three main themes (Figure 1). Namely, the program's approach, the home support setting and views around physical activity and older people. "Delivering care in a crisis" was further identified, due to the impact of the COVID-19 pandemic which crossed the study's timeline. Finally, participants shared practical suggestions and opportunities for the future direction of embedding physical activity within formal care services.

\section{The Program's Approach}

Overall, there was a positive perception of the program's "fit" within the home support environment, the focus on "movement prompts" and "motivators" and the structured training provided to staff.

\section{The Program "Fit" Within Home Care Structures}

CTM was considered a relatively practical approach to embed into the daily routine of home support visits. "When you know the steps and the routine, [CTM] becomes very easy" HCA007. The CTM method of incorporating the intervention into the clients' routine was viewed as central to increasing daily levels of activity. "If you're going to implement it into their daily routine, [clients] don't even know that they're doing [CTM]" HCA024. 


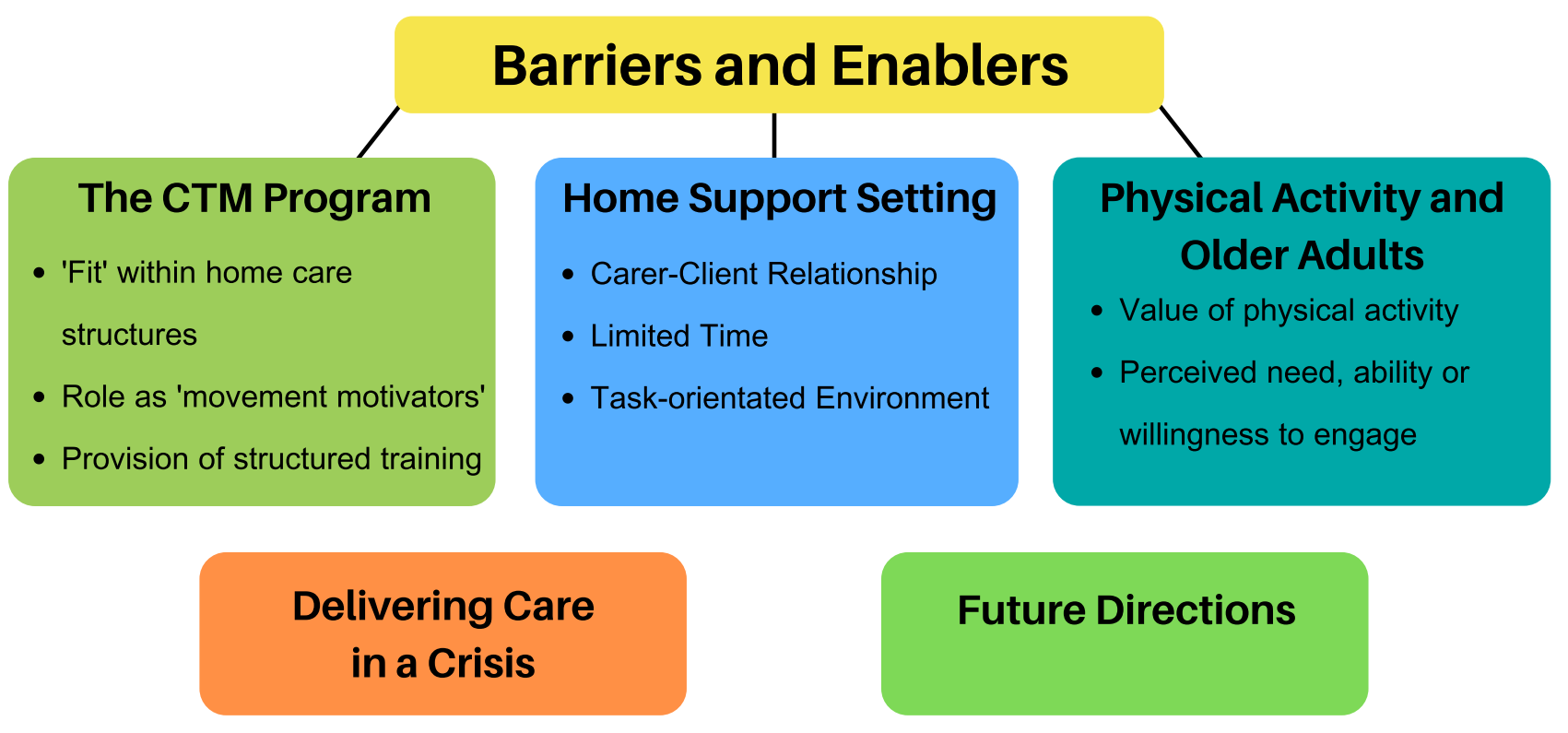

Figure I Factors affecting the delivery of the Care to Move approach within formal care services.

\section{Role as "Movement Motivators"}

The HCAs role as "movement motivators", was viewed as a positive concept that encouraged older adults to participate in the program. "A lot of [clients] sit down all day and they need someone to lead them" HCA029. HCAs perceived an ability to disrupt periods of sedentary behavior through the application of movement prompts "Like a lot of people when you go in, they are just sitting on the chair doing nothing. ... if you can get them up, if you can motivate them, it's great." HCA021. Some HCAs discerned the difference between physical activity and exercise, and their intended benefits. "You don't say exercise because it's not really, it's about promoting movement you know?" HCA024. However, many HCAs used "movement" and "exercises" interchangeably throughout the interviews.

\section{Provision of Structured Staff Training}

The provision of the structured training prior to CTM delivery was largely viewed as sufficient and practical. "I thought it was a very good course ... for the 2 days ... there was loads in it that you are able to do" HCA020. Although, a need for additional on the ground support throughout the program was also identified "I think it would be good to have someone that you can ring ...... even call in and give us an idea of what to do" HCA010 and further commented that "... we are not qualified to do physio even though we do help some of the older people out" HCA010. The applicability of "movement prompts" was again reflected, as well as practical aspects such as employing a "stand-to-move" approach, where unaided standing is promoted with prompts on foot placement.

Before I was doing Care to Move ... I was actually helping everybody to stand up with the help of the crutch or the frame, with one hand under the arm ... Just to give them a hand, it is definitely way better to stand to move HCA007.

\section{The Home Support Setting}

The potential for relationship building and interaction with clients was identified, however, the "time and task nature of home support" was a recurring challenge in embedding physical activity within the current formal support structures.

\section{The Carer-Client Relationship}

A persistent thread across all interviews was the value of the relationship between the HCA and the client. "It makes me feel good knowing that I'm helping her" HCA023. For some HCAs, their relationship with the client, was perceived to enable personalizing the delivery of CTM. "I know how far to push with one and then how far to push with the other" HCA022 and "Sometimes she says 'oh I'm so tired, I can't do it today" and I say "that's ok do not worry we will leave 
it ... you do not say you have to do [CTM]" HCA029. Others observed an opportunity in CTM to increase interaction with the client. "If [clients are] willing to participate in [CTM], it would be great for them because it's interaction with them" HCA024 and "I took part when they were doing [CTM]. I was doing [CTM] with them" HCA008.

\section{Limited Time}

The limited-time available to HCAs to complete the existing essential tasks was viewed as a key and recurrent barrier to program delivery.

You probably get a half an hour call which is at lunchtime but by the time you go in and make the lunch, sure the half is up ... it can be a bit difficult to try and fit them exercises in as well HCA005.

Workload issues were described as an ongoing challenge within home support services, irrespective of the delivery of the intervention. "You don't actually have the time even to be doing what you're doing, do you know what I mean?" HCA003. The burden of the research study paperwork (aimed at capturing daily goals of the intervention) was noted to impact further on an already time-limited visit. "You barely get time to fill in your daily log and what you [have] done with that client today without doing all that paperwork". HCA002 and " ... you haven't got time to be doing all of that [paperwork]" HCA003.

\section{Task-Orientated Environment}

The task-orientated nature of home support services emerged as a barrier to delivery, where assistance with activities of daily living were a priority over the intervention.

It can be difficult if you're getting them out of bed and you're getting them washed and you're getting them dressed and you're giving them their breakfast. Like it does take up your whole hour. Even a little bit of an extra on to that hour HCA005.

This task focused method of care delivery combined with limited time was frequently cited in home visits with personal care duties. "Some clients, you wouldn't have a minute to yourself like, you could be getting them washed and dressed, you wouldn't have a minute" HCA003.

\section{Physical Activity and the Older Adult}

Both positive and negative perceptions of the role of physical activity in older adults were evident. Some perceived value in enabling physical activity, while other comments broadly suggested that older adults "couldnot, would not, or should not' engage in physical activity, ie, due to refusal, poor functional ability or that this activity was not suitable for adults of more advanced age. ${ }^{32,33}$

\section{Perceived Value of Physical Activity for Older Adults}

Many HCAs reported a positive attitude towards the approach and viewed that it translated into improvements in physical function and activity among older adults.

I can see the benefits of [CTM] and it makes me feel good knowing that I'm helping her and that she is happy and confident then because she is more active and she is moving more muscles, working her body the way she should be HCA023.

HCAs further highlighted buy-in from the service users and positive behavior change in response to the intervention. "The minute I go in she'll say 'are we doing the exercises?" HCA005 and "[Clients] used to say we have got to do a little bit of movement today" HCA008. An understanding of the importance of physical activity and movement for older adults was noted "[HCAs] are doing [CTM] to get the older people moving so that it helps them with their stiffness and keeps them going" HCA010.

\section{Older Adults Need, Ability or Willingness to Engage in Physical Activity}

HCAs identified the clients' willingness to engage in the physical activity approach as central to the success of the program. "Care to Move can be the carer's duty but it really depends on them, it really depends on the clients" HCA006. 
However, others commented on clients' reluctance to participate in the intervention. Reasons for this lack of engagement were perceived to be lack of interest, dislike of "being told what to do" or fatigue.

You are talking about probably 70 or 80 -year old's, and taking them up and down on the chair, moving their feet, and some people did feel it a bit tiresome to keep asking an 80-year old to have to do that HCA002.

Other subthemes identified may reflect negative perceptions around physical activity in older age, for example, the need or ability of older adults to partake due to advanced age, dementia, co-morbidities, or frailty. Some HCA, however, commented on the perceived risks, such as causing injury or pain.

\section{Delivering Care in a Crisis - Impact of the COVID-19 Pandemic}

The study interviews were conducted between August-September 2020, and therefore the impact of the COVID-19 pandemic on healthcare was reflected throughout the interviews. The HCAs described completing only essential tasks and keeping visits as short as possible. "I was only bringing her shopping, so I wasn't able to do any of [CTM] at all ... I couldn't do anything there. I was just in and out" HCA020. The loss of physical activity, an increase in anxiety and a general decline in physical and cognitive function of the older adults during COVID-19 was widely described "[Clients] were afraid and they haven't been going out and getting as much exercise" HCA023 and "When COVID started happening that's when we kind of stopped [CTM] because she had other worries on her mind" HCA024.

She lives on a top floor apartment ... she was using the stairs but now she's not using them, so she has really slowed down, so I just take her for a walk out onto the landing HCA001 and "She was isolated and wasn't going out ... she was absolutely isolated" HCA001.

\section{Future Directions}

During the interviews, HCAs were asked to offer suggestions for delivering the program within home support services and to share their views on the potential role of technology. Given that the most cited barrier was limited time, HCAs proposed that additional time should be allocated to deliver this specific initiative within each home support visit. Other opportunities for future improvement included full workforce training in the program and designated CTM program "champions" or support personnel.

\section{Embed the Program into Formal Workforce Training}

Participants reported that CTM training should be delivered to all HCA employees and embedded into workforce training.

I think everyone should do [CTM]. I think it is essential for everyone to take part in it. It's not just like select people, it would be great if everyone did it at the start ... I think they would see a lot of results from [CTM] if everyone did it HCA024.

HCAs described an opportunity to integrate CTM training into the mandatory Care Skills modules required for their role. "I think going forward that [CTM] definitely should be part of a module rather than a training, that shows the benefits if you get in at the right time" HCA026.

\section{Designate Champions of the Program}

HCAs commented on the potential benefits of having designated local CTM coordinators or advisors within their organization.

You know, if you were kind of stuck and say 'well I do this exercise but she's finding this exercise more difficult than the rest. Is there an easier way or another way that we could do it?' HCA005.

"It would be great to have someone to have a chat with about [CTM]" HCA024. Others suggested that a designated person may have a potential role in client motivation. "It might help when you have some clients who do not want to do 
[CTM], you know having someone else there to try motivate them" HCA027. This aligns with earlier views of a perceived lack of on the ground support, as a potential barrier.

\section{The Role of Technology}

In response to the ongoing COVID-19 pandemic, HCAs were asked about the potential of using technology to assist with delivering the programme. HCAs did not perceive technology readiness among most of their clients. "Well, most of them wouldn't have a phone like they wouldn't be able to do much because they don't know what technology is." HCA023 and "They can't even turn their telly [television] on or use the remote control." HCA022. HCAs perceived that being familiar with, or owning a smartphone or computer was viewed as an exception among their clients, and potentially relevant to one client in their full caseload (not specifically in the study). "Yeah, like one comes to mind, she has a phone, and she uses WhatsApp so she is the only one that would have an idea of what Zoom is and all of that." HCA023, "One has the laptop and a mobile phone" HCA006 and "One can. Just the one. She can go on to Facebook and she can write a message on her touchscreen and, emails and all that she wouldn't be able to do that." HCA025. Notably, the absence of technology proficiency was also reflected among the HCAs. "I can't even turn on a computer" HCA010 and "I can answer, send a text message and hang up and that's about it. I have it all on my phone, but I can't use it. I don't even know how to send an email" HCA022.

\section{Discussion}

We investigated the feasibility of delivering a physical activity program "Care to Move" (CTM) to older dependent adults through existing home support services. National and international policies support both strategies for aging in place and the benefits of physical activity among all older populations. ${ }^{34,35}$ The present qualitative study aimed to explore the experience and perceptions of HCAs who were trained in and delivered the program. The overall goal was to better understand the feasibility of translating this initiative into practice and to move home support beyond its traditional taskbased focus. Barriers and enablers to the delivery of the intervention were identified across three main themes. Namely, 1) the program, 2) the home support setting, and 3) physical activity for older adults. Enablers included the "fit" of the CTM approach, the role of movement motivators, provision of structured training, carer-client relationship, and the perceived value of physical activity for older adults. Recurring barriers included limited-time, the task-orientated nature of home support services and the older adults' need, ability, or willingness to engage in physical activity. 4) "Delivering care in a crisis", reflecting the ongoing Covid-19 pandemic and 5) "Future directions" outlining practical suggestions shared by HCAs are further noted.

With respect to the program, the qualitative evidence indicated that the CTM approach was a good "fit" within the home support setting, where the HCAs' role was to encourage physical activity through movement prompts. More strenuous activities are less likely to be feasible in this group, because of the prevalence of fatigue and deconditioning, and a program of activities that focusses instead on reducing sedentary behavior may be more appropriate. The findings indicated that older adults partook in the movement activities with the HCA, however, it was unclear the degree to which this may have translated into increased activity between visits. Although CTM is not an exercise program, many HCAs used the terms "movement" and "exercise" interchangeably throughout the interviews, however there was no indication that this affected implementation. It was generally perceived that the program was adequately supported by staff training at onset, though more on the ground support or local "champions", within the organization, for the program was suggested. The time-limited and task-orientated nature of home support were cited as recurring barriers to embedding the program within home support. Given the time constraints and competing tasks, the necessity to prioritize assistance with ADLs, specifically personal care, over promoting physical activity was clearly articulated. Ideally, future work would examine the feasibility of embedding physical activity initiatives and prompts within the formal care plan. Previous studies, for example, have identified collaborative goal setting and personalised support plans as central to the implementation of restorative home care interventions. ${ }^{36}$

Importantly, many HCAs perceived the program as having practical benefits for their clients, for example in maintaining mobility, and in relationship-building. However, this was not universal, with a view identified suggesting that older adults "could not, would not or should not", engage in physical activity. This was attributed to several reasons 
including reduced ability (ie due to impaired physical or cognitive function), unwillingness or resistance to partake and a perception that older adults ought not to participate in physical activity. The latter, may reflect negative perceptions around physical activity in aging which have been similarly reported elsewhere, ${ }^{37,38}$ often reflecting views that older people should "rest". While evidence to the contrary is now vast, and physical inactivity is strongly associated with muscle loss (sarcopenia) ${ }^{39,40}$ and poorer physical and mental health outcomes. ${ }^{38}$ Current guidelines recommend physical activity for all older adults, including people living with frailty or disability. ${ }^{35}$ The findings of the present study highlight an opportunity for further awareness-raising as part of the standardized HCA training to highlight the role of physical activity for adults aged $80+$ years, living with frailty or disability. The present study, however, cannot discern the degree to which the HCAs own values and views on physical activity in general impacted their perceptions in the study. The practical risk of inducing pain or injury while administering the program was noted, though interesting falls risk was not specifically mentioned, in contrast to findings in acute-based settings. ${ }^{32,33}$

The original study did not set out to specifically investigate the impact of the COVID-19 pandemic on the intervention. Given that the study crossed the timeline of the pandemic, the impact of delivering care in a crisis was evident throughout interviews. There was consistent reporting of high levels of physical decline among the older adults, fear and isolation and challenges in delivering home support. These findings mirror the national and international evidence on COVID-19.41,42 The pandemic generated heightened interest in the potential role of using technology (smartphones, tablets, videocalls) in supporting older populations. ${ }^{43}$ Our findings suggest that technology readiness was the exception in this older cohort. Notably, the HCAs viewed the older adults and their own ability to use technology as poor. This could be attributed in part, to the high prevalence of cognitive impairment and possibly vision or hearing impairments in this age group. ${ }^{6,43}$ The HCA views may be a confounder, and input from the older adults or their family members would be required to give further context.

The present study suggests that promoting physical activity among older adults through formal home support services is feasible, but implementation would require changes in the home support structure and the workforce (Table 1). There is growing evidence of reablement, reactivation, rehabilitation, and restorative (4R) interventions within home care services however, many are short-term initiatives and delivered by interdisciplinary teams, while few have examined the experience of HCAs delivering these approaches. ${ }^{36} \mathrm{~A}$ recent systematic review of physical activity programs for older people within home care services highlighted a need for further research around factors that influence adherence and effectiveness. ${ }^{44}$ Evidence to support such initiatives is strong, the benefits of physical activity in aging are consistent, physical activity is the treatment "prescription" for age-related muscle loss (sarcopenia) and current guidelines recommend physical activity for older people, including people with disability and frailty. ${ }^{15,16}$ The initiative is in line with policies nationally and internationally to support aging in place, and with the desire of older people to live at home in their community. The current estimated 23.9 million care hours delivered annually in Ireland offers opportunities to move to more personalised supports, including promoting physical activity. ${ }^{4}$ This is timely in Ireland, as the Department of Health is currently developing a statutory scheme for the financing and regulation of home support services. ${ }^{45}$

This study has several strengths and limitations. To the best of the authors' knowledge this is the first study to examine the experience of HCAs embedding physical activity within home support services in Ireland. The CTM approach was designed to encourage older adults to "move more", rather than as a structured exercise program. The HCAs interviewed in this study represented an experienced workforce in the care of older people. Limitations must be noted, the use of close-ended interview questions may have impacted the ability to explore related ideas and provide appropriate depth however, the interview guide was developed based on previous research. ${ }^{25}$ As a feasibility study and compounded by COVID-19, the sample size was relatively small, interviews with HCAs were conducted by phone rather than face to face, and perceptions may be unintentionally influenced by the broader challenges in delivering communitycare at the time. Nonetheless, consistent with previous studies, our findings suggest embedding physical activity initiatives within home support services is feasible but challenges remain. ${ }^{25,44}$

\section{Conclusion}

The present study examined the experience of HCAs delivering a home-based physical activity program. The findings suggest that embedding physical activity initiatives within home support services is feasible. Overall, there was a positive 
Table I Learnings for Embedding Physical Activity Within Formal Support Services for Community Dwelling Older Adults Based on HCAs Experience

\begin{tabular}{|l|l|l|}
\hline Theme & Summary of Findings & Considerations at Organizational and Policy Level \\
\hline $\begin{array}{l}\text { The physical activity } \\
\text { program }\end{array}$ & $\begin{array}{l}\text { The CTM approach appeared to be a good fit within home support } \\
\text { services } \\
\text { The staff training was practical and of sufficient duration, but additional } \\
\text { supports were suggested. }\end{array}$ & $\begin{array}{l}\text { - A program that encourages older people to "move more and sit less", appears to fit with home } \\
\text { support services for community-dwelling frail older people } \\
\text { - Provide initial staff training. } \\
\text { - Embed the training across the full workforce } \\
\text { - Provide additional on the ground staff supports (leaders, champions) }\end{array}$ \\
\hline $\begin{array}{l}\text { The home support } \\
\text { setting }\end{array}$ & $\begin{array}{l}\text { The time and task-oriented routine of home support were challenges } \\
\text { to embedding the initiative into services. }\end{array}$ & $\begin{array}{l}\text { - Explore the feasibility of prescribing "movement prompts" in the care plans for older adults } \\
\text { receiving home support } \\
\text { - Review the time needs and costs for care visits with physical activity initiatives } \\
\text { - A system change to reconfigure home support beyond a "task" based nature to more broadly } \\
\text { support health, well-being, and quality of life approaches }\end{array}$ \\
\hline $\begin{array}{l}\text { Physical activity and } \\
\text { older adults }\end{array}$ & $\begin{array}{l}\text { Positive and negative perceptions of the value of physical activity for } \\
\text { older frail adults were identified }\end{array}$ & $\begin{array}{l}\text { - Awareness raising for staff and more widely, around the role and benefits of physical activity in frail } \\
\text { older people }\end{array}$ \\
\hline
\end{tabular}


perception of the programs "fit" within home support services and perceived value and benefit of physical activity for older adults. Restructuring of services review of time needs, engaging HCAs, and moving beyond traditional "task orientated" care models to more personalised proactive approaches may facilitate this initiative and support aging in place.

\section{Data Sharing Statement}

Data supporting the results reported in this manuscript is available upon request. Interested persons may contact the corresponding author.

\section{Ethical Approval and Consent to Participate}

This study was approved by the Royal College of Surgeons in Ireland Research Ethics Committee (REC - 2018:1489), and informed consent was obtained from all participants.

\section{Acknowledgments}

The authors would like to thank the staff who delivered the CTM program and who participated in the interviews for this study.

\section{Funding}

This work was funded by an APA-2017-013 Health Research Board of Ireland Applied Partnership Award.

\section{Disclosure}

Bex Townley and Dawn A. Skelton are Directors of Later Life Training Ltd, a not-for-profit company delivering training in exercise delivery with older people, including the Care to Move program. BT and DAS had no role in the design of the interview questions, in conducting the interviews or in the analysis of the qualitative data. BT and DAS had no role in the design of the interview questions (designed by FH, LS, MOS), in conducting the interviews (conducted by FH, LS) or in the analysis of the qualitative data (conducted by LS, MOS, AW). DAS and BT report personal fees from Later Life Training Ltd, during the conduct of the study; personal fees from Later Life Training Ltd, outside the submitted work. Debbie Rooney is CEO of North Dublin Home Care and Lisa Murphy is the Service and Quality Manager at North Dublin Home Care. North Dublin Homecare provide a PhD funding bursary for author LS and had no role in the design of the study; in the collection, analyses, or interpretation of data; in the writing of the manuscript, or in the decision to publish the results. The aforementioned authors report no other potential conflicts of interest in this work. All other authors report no conflicts of interest in this work.

\section{References}

1. United Nations. World population ageing report 2015; 2015.

2. Giri S, Chenn LM, Romero-Ortuno R. Nursing homes during the COVID-19 pandemic: a scoping review of challenges and responses. Eur Geriatr Med. 2021;12(6):1127-1136. doi:10.1007/s41999-021-00531-2

3. Wiles JL, Leibing A, Guberman N, Reeve J, Allen RES. The meaning of "aging in place" to older people. Gerontologist. 2012;52(3):357-366. doi:10.1093/geront/gnr098

4. Health Service Executive. National service plan 2021; 2021. Available from: https://www.hse.ie/eng/services/publications/serviceplans/nationalservice-plan-2021.pdf. Accessed February 23, 2022.

5. Murphy CM, Whelan BJ, Normand C. Formal home-care utilisation by older adults in Ireland: evidence from the Irish longitudinal study on ageing (TILDA). Health Soc Care Community. 2015;23(4):408-418. doi:10.1111/hsc.12157

6. Aspell N, O'Sullivan M, O’Shea E, et al. Predicting admission to long-term care and mortality among community-based, dependent older people in Ireland. Int J Geriatr Psychiatry. 2019;34(7):999-1007. doi:10.1002/gps.5101

7. O'Shea E, Monaghan C. An economic analysis of a community-based model for dementia care in Ireland: a balance of care approach. Int Psychogeriatr. 2017;29(7):1175-1184. doi:10.1017/S1041610217000400

8. Kelly S, O’Brien I, Smuts K, O’Sullivan M, Warters A. Prevalence of frailty among community dwelling older adults in receipt of low level home support: a cross-sectional analysis of the North Dublin Cohort. BMC Geriatr. 2017;17:121. doi:10.1186/s12877-017-0508-2

9. O’Brien I, Smuts K, Fan CW, O'Sullivan M, Warters A. High prevalence of dementia among community dwelling older adults in receipt of state funded home care packages: implications for health care planning. Irish J Psychol Med. 2017;34(1):1-6. doi:10.1017/ipm.2017.80 
10. Rockwood K, Song X, MacKnight C, et al. A global clinical measure of fitness and frailty in elderly people. CMAJ. 2005;173(5):489-495. doi:10.1503/cmaj.050051

11. Harvey JA, Chastin SFM, Skelton DA. How sedentary are older people? A systematic review of the amount of sedentary behavior. $J$ Aging Phys Act. 2015;23(3):471-487. doi:10.1123/japa.2014-0164

12. Harvey JA, Chastin SFM, Skelton DA. Breaking sedentary behaviour has the potential to increase/ maintain function in frail older adults. $J$ Frailty Sarcopenia Falls. 2018;3(1):26-31. doi:10.22540/JFSF-03-026

13. Liu CK, Fielding RA. Exercise as an intervention for frailty. Clin Geriatr Med. 2011;27(1):101-110. doi:10.1016/j.cger.2010.08.001

14. Oliveira JS, Pinheiro MB, Fairhall N, et al. Evidence on physical activity and the prevention of frailty and sarcopenia among older people: a systematic review to inform the world health organization physical activity guidelines. J Phys Act Health. 2020;17(12):1247-1258. doi:10.1123/ jpah.2020-0323

15. Izquierdo M, Merchant RA, Morley JE, et al. International exercise recommendations in older adults (ICFSR): expert consensus guidelines. $J$ Nutr Health Aging. 2021;25(7):824-853. doi:10.1007/s12603-021-1665-8

16. UK CMO's Physical Activity Guidelines. Physical activity guidelines: UK chief medical officers' report. Department of Health and Social Care; 2019. Available from: https://www-gov-uk.elib.tcd.ie/government/publications/physical-activity-guidelines-uk-chief-medical-officers-report. Accessed November 17, 2021.

17. Füzéki E, Engeroff T, Banzer W. Health benefits of light-intensity physical activity: a systematic review of accelerometer data of the National Health And Nutrition Examination Survey (NHANES). Sports Med. 2017;47(9):1769-1793. doi:10.1007/s40279-017-0724-0

18. Taylor J, Walsh S, Kwok W, et al. A scoping review of physical activity interventions for older adults. Int J Behav Nutr Phys Act. 2021;18(1):82. doi:10.1186/s12966-021-01140-9

19. Moschny A, Platen P, Klaaßen-Mielke R, Trampisch U, Hinrichs T. Barriers to physical activity in older adults in Germany: a cross-sectional study. Int J Behav Nutr Phys Act. 2011;8(1):121. doi:10.1186/1479-5868-8-121

20. Burton E, Lewin G, Boldy D. Physical activity preferences of older home care clients. Int J Older People Nurs. 2015;10(3):170-178. doi:10.1111/ opn. 12065

21. Bjerk M, Brovold T, Davis JC, Skelton DA, Bergland A. Health-related quality of life in home care recipients after a falls prevention intervention: a 6-month follow-up. Eur J Public Health. 2020;30(1):64-69. doi:10.1093/eurpub/ckz106

22. Gill TM, Baker DI, Gottschalk M, Peduzzi PN, Allore H, Byers A. A program to prevent functional decline in physically frail, elderly persons who live at home. $N$ Engl J Med. 2002;347(14):1068-1074. doi:10.1056/NEJMoa020423

23. Weber M, Belala N, Clemson L, et al. Feasibility and effectiveness of intervention programmes integrating functional exercise into daily life of older adults: a systematic review. Gerontology. 2018;64(2):172-187. doi:10.1159/000479965

24. Home and Community Care Ireland. Home and community care Ireland calls for regulation in the home care industry. Home and Community Care Ireland; 2018. Available from: https://hcci.ie/home-and-community-care-ireland-calls-for-regulation-in-the-home-care-industry/. Accessed January 4, 2022.

25. Burton E, Boyle EJ, O’Connell H, Lewin G, Petrich M, Hill KD. Community care staff attitudes towards delivering a falls prevention exercise intervention to community care clients. Health Soc Care Community. 2021;29(2):416-424. doi:10.1111/hsc.13101

26. Horgan NF, Cummins V, Doyle F, et al. Enhancing existing formal home care to improve and maintain functional status in older adults: protocol for a feasibility study on the implementation of the Care to Move (CTM) programme in an Irish healthcare setting. J Frailty Sarcopenia Falls. 2020;5 (1):10-16. doi:10.22540/JFSF-05-010

27. Later Life Training. About Care to Move (CTM). Later life training; 2021. Available from: https://www.laterlifetraining.co.uk/courses/care-movectm-course/. Accessed November 2, 2021.

28. Lambert VA, Lambert CE. Qualitative descriptive research: an acceptable design. Pac Rim Int J Nurs Res. 2012;16(4):255-256.

29. Braun V, Clarke V. Using thematic analysis in psychology. Qual Res Psychol. 2006;3(2):77-101. doi:10.1191/1478088706qp063oa

30. Braun V, Clarke V. Reflecting on reflexive thematic analysis. Qual Res Sport Exerc Health. 2019;11(4):589-597. doi:10.1080/ 2159676X.2019.1628806

31. Braun V, Clarke V. Successful Qualitative Research: A Practical Guide for Beginners. SAGE; 2013.

32. Lim SH, Ang SY, Ong HK, et al. Promotion of mobility among hospitalised older adults: an exploratory study on perceptions of patients, carers and nurses. Geriatr Nurs. 2020;41(5):608-614. doi:10.1016/j.gerinurse.2020.03.015

33. Dermody G, Kovach CR. Barriers to promoting mobility in hospitalized older adults. Res Gerontol Nurs. 2018;11(1):17-27. doi:10.3928/ 19404921-20171023-01

34. Bull FC, Al-Ansari SS, Biddle S, et al. World Health Organization 2020 guidelines on physical activity and sedentary behaviour. Br J Sports Med. 2020;54(24):1451-1462. doi:10.1136/bjsports-2020-102955

35. Health Service Executive. The national guidelines on physical activity for Ireland; 2009. Available from: https://www.hse.ie/eng/about/who/ healthwellbeing/our-priority-programmes/heal/heal-docs/the-national-guidelines-on-physical-activity-for-ireland.pdf. Accessed February 23, 2022.

36. Sims-Gould J, Tong CE, Wallis-Mayer L, Ashe MC. Reablement, reactivation, rehabilitation and restorative interventions with older adults in receipt of home care: a systematic review. J Am Med Dir Assoc. 2017;18(8):653-663. doi:10.1016/j.jamda.2016.12.070

37. Andrews RM, Tan EJ, Varma VR, et al. Positive aging expectations are associated with physical activity among urban-dwelling older adults. Gerontologist. 2017;57(suppl_2):S178-S186. doi:10.1093/geront/gnx060

38. Cunningham C, O’ Sullivan R, Caserotti P, Tully MA. Consequences of physical inactivity in older adults: a systematic review of reviews and meta-analyses. Scand J Med Sci Sports. 2020;30(5):816-827. doi:10.1111/sms.13616

39. Cruz-Jentoft AJ, Bahat G, Bauer J, et al. Sarcopenia: revised European consensus on definition and diagnosis. Age Ageing. 2019;48(1):16-31. doi:10.1093/ageing/afy169

40. Swan L, Warters A, O’Sullivan M. Socioeconomic inequality and risk of sarcopenia in community-dwelling older adults. Clin Interv Aging. 2021;16:1119-1129. doi:10.2147/CIA.S310774

41. Bailey L, Ward M, DiCosimo A, et al. Physical and mental health of older people while cocooning during the COVID-19 pandemic. QJM Mon J Assoc Physicians. 2021:hcab015. doi:10.1093/qjmed/hcab015

42. Ward P, O’Mahoney P, Kenny RA. Altered lives in a time of crisis: the impact of the Covid-19 pandemic on the lives of older adults in Ireland. Findings from the Irish longitudinal study on ageing; 2021. Available from: https://tilda.tcd.ie/publications/reports/pdf/c19-key-findings-report /COVID-19\%20Key\%20Findings\%20Report.pdf. Accessed November 15, 2021. 
43. Lam K, Lu AD, Shi Y, Covinsky KE. Assessing telemedicine unreadiness among older adults in the United States during the COVID-19 pandemic. JAMA Intern Med. 2020;180(10):1389. doi:10.1001/jamainternmed.2020.2671

44. Burton E, Farrier K, Galvin R, et al. Physical activity programs for older people in the community receiving home care services: systematic review and meta-analysis. Clin Interv Aging. 2019;14:1045-1064. doi:10.2147/CIA.S205019

45. Walsh B, Lyons S. Demand for the statutory home support scheme; 2021. Available from: https://www.esri.ie/publications/demand-for-the-statutory -home-care-scheme. Accessed November 15, 2021.

\section{Publish your work in this journal}

Clinical Interventions in Aging is an international, peer-reviewed journal focusing on evidence-based reports on the value or lack thereof of treatments intended to prevent or delay the onset of maladaptive correlates of aging in human beings. This journal is indexed on PubMed Central, MedLine, CAS, Scopus and the Elsevier Bibliographic databases. The manuscript management system is completely online and includes a very quick and fair peer-review system, which is all easy to use. Visit http://www.dovepress.com/testimonials.php to read real quotes from published authors.

Submit your manuscript here: https://www.dovepress.com/clinical-interventions-in-aging-journal 